\title{
Nuclear incompressibility in the quasilocal density functional theory
}

\author{
V. B. Soubbotin and V. I. Tselyaev \\ Nuclear Physics Department, V. A. Fock Institute of Physics, St. Petersburg State University, 198504, St. Petersburg, Russia \\ X. Viñas \\ Departament d'Estructura i Constituents de la Matèria, Facultat de Física, Universitat de Barcelona, \\ Diagonal 645 E-08028 Barcelona, Spain
}

(Received 9 March 2004; published 14 June 2004)

\begin{abstract}
We explore the ability of the recently established quasilocal density functional theory for describing the isoscalar giant monopole resonance. Within this theory we use the scaling approach and perform constrained calculations for obtaining the cubic and inverse energy weighted moments (sum rules) of the RPA strength. The meaning of the sum rule approach in this case is discussed. Numerical calculations are carried out using Gogny forces and an excellent agreement is found with $\mathrm{HF}+\mathrm{RPA}$ results previously reported in literature. The nuclear matter compression modulus predicted in our model lies in the range 210-230 MeV which agrees with earlier findings. The information provided by the sum rule approach in the case of nuclei near the neutron drip line is also discussed.
\end{abstract}

DOI: 10.1103/PhysRevC.69.064312

PACS number(s): 21.60.Jz, 31.15.Ew, 31.15.Gy

\section{INTRODUCTION}

Recently we have established the quasilocal density functional theory (QLDFT) and its application for describing the nuclear ground state properties [1]. It is just a generalization of the local Hohenberg-Kohn-Sham (HKS) theory $[2,3]$ and is based on the definition of an universal energy density functional $\mathcal{E}$ of the Slater density matrix (DM) $\rho_{0}$ [1]. One can define the quasilocal energy functional $E[\hat{n}, \hat{\tau}, \hat{\mathbf{J}}]$ through a many-to-one mapping of the density matrix $\rho_{0}$ to a set of local quantities $\hat{n} \equiv\left\{n_{p}, n_{n}\right\}, \hat{\tau} \equiv\left\{\tau_{p}, \tau_{n}\right\}, \hat{\mathbf{J}} \equiv\left\{\mathbf{J}_{p}, \mathbf{J}_{n}\right\}$, where $n_{q}, \tau_{q}, \mathbf{J}_{q}$ are the local, the kinetic energy, and the spin densities of each kind of nucleons $(q=p, n)$ :

$$
E[\hat{n}, \hat{\tau}, \hat{\mathbf{J}}]=\inf _{\rho_{0} \rightarrow \hat{n}, \hat{\tau}, \hat{\mathbf{J}}} \mathcal{E}\left[\rho_{0}\right] .
$$

The main property of the functional (1) is that its minimum provides the exact ground state energy, which is attained at the true local nucleon densities $\hat{n}$. It is worthwhile to note that in this case the equilibrium $\hat{\tau}$ and $\hat{\mathbf{J}}$ densities are not the exact ones and they correspond to the system without correlations.

The functional $E[\hat{n}, \hat{\tau}, \hat{\mathbf{J}}]$ consists of two terms:

$$
E[\hat{n}, \hat{\tau}, \hat{\mathbf{J}}]=E_{0}[\hat{n}, \hat{\tau}, \hat{\mathbf{J}}]+E_{\mathrm{RC}}[\hat{n}],
$$

where $E_{0}[\hat{n}, \hat{\tau}, \hat{\mathbf{J}}]$ is a Hartree-Fock (HF) energy functional reduced to the quasilocal form, and $E_{\mathrm{RC}}$ is the residual correlation energy. The HF contribution corresponds to a finiterange density-independent effective force. Its quasilocal form is calculated using the extended Thomas-Fermi theory [4], and consists of a kinetic energy part, a Hartree term, a local exchange contribution, and a spin-orbit energy as used in the Skyrme energy functionals [1]. To compute this HF functional we use the density-independent finite-range part of the Gogny force [5]. The main formulas for $E_{0}$ are re- ported in Ref. [1]. The residual correlation energy $E_{\mathrm{RC}}[\hat{n}]$ is taken phenomenologically and is parametrized similar to the contribution of the density-dependent part of the Skyrme or the Gogny interactions.

The aim of the QLDFT is to describe properties of the nuclear ground state such as the total energy $E_{\text {g.s. }}$, the local particle densities $\hat{n}$, the neutron and proton separation energies (chemical potentials) of double magic nuclei. To do that use is made of the variational principle to obtain a set of single-particle equations similar to the Kohn-Sham ones but containing additionally a position-dependent effective mass and a spin-orbit potential [1]. We have used this quasilocal approach to obtain some nuclear ground-state properties of several magic nuclei with the Gogny D1S force [6]. We have found a very good agreement between the binding energies and root mean square radii computed using the QLDFT and the corresponding values calculated with the full HF method [1].

Now we are in turn to investigate if the QLDFT is also able to predict some information about excited nuclear states. In this paper we want to analyze the collective breathing mode and to study the isoscalar giant monopole resonance (ISGMR) in finite nuclei using our model. The experimental value of the excitation energy of the ISGMR is mainly extracted from the analysis of the inelastic $\left(\alpha, \alpha^{\prime}\right)$ scattering data (see Ref. [7] and references therein). From the experimental excitation energies in medium and heavy nuclei it is possible to estimate the nuclear matter compression modulus $K_{\infty}$. In particular, self-consistent HF plus random phase approximation (RPA) calculations using Gogny [8] or Skyrme forces [9] determine $K_{\infty}$ to be $210-230 \mathrm{MeV}$.

\section{THE BASIC THEORY}

Giant resonances are understood in terms of small amplitude oscillations of nuclei as a response to an external field generated by electromagnetic or hadronic probes. The most 
widely used theoretical framework for describing these vibrations is the RPA [10] which allows to obtain the strength function $S(E)$ that measures the nuclear response. For medium and heavy nuclei far from the drip line, the strength $S(E)$ corresponding to the breathing mode is mainly concentrated in a rather narrow region of the energy spectrum. In these cases the knowledge of a few low energy moments of $S(E)$ (sum rules), which are defined as

$$
m_{k}=\sum_{\nu \neq 0}\left(E_{\nu}-E_{0}\right)^{k}|\langle\nu|Q| 0\rangle|^{2},
$$

where $Q=\Sigma_{i} r_{i}^{2}$ is the one-body monopole excitation operator, can provide a useful information on the average properties of the ISGMR. If $k$ is an odd integer, the sum rules $m_{k}$ can be written as expectation values of some commutators calculated in the exact ground state [11]. For example,

$$
m_{1}=\langle 0|[Q,[H, Q]]| 0\rangle
$$

and

$$
m_{3}=\langle 0|[[Q, H],[H,[H, Q]]]| 0\rangle .
$$

From these moments one can estimate several average energies as

$$
\bar{E}_{k, k-2}=\sqrt{\frac{m_{k}}{m_{k-2}}} .
$$

The full quantal calculation of the sum rules is still a complicated task because the exact ground state wave function is usually unknown. However, if the energy moments of $S(E)$ are evaluated at $1 p 1 h$ (RPA) level, it is possible to replace the exact ground-state wave functions by the uncorrelated $\mathrm{HF}$ ones in the calculation of the sum rules.

For forces that commute with the monopole excitation operator $Q$, as it happens for the Skyrme and Gogny interactions, the only contribution to the commutator $[Q,[H, Q]]$ comes from the kinetic energy part of the Hamiltonian. Thus the $m_{1}$ sum rule is given by (see Ref. [11])

$$
m_{1}=\frac{2 \hbar^{2}}{M} A\left\langle r^{2}\right\rangle_{0},
$$

where the expectation value of the operator $r^{2}$ is calculated with the HF wave functions.

The direct evaluation of the commutators entering the formula (5) for $m_{3}$ is a rather cumbersome task which can be avoided computing the $m_{3}$ sum rule of the RPA monopole strength function through the scaling method [11]. Using the scaled ground-state wave function

$$
\Psi_{\eta}\left(\mathbf{r}_{1}, \ldots, \mathbf{r}_{A}\right)=\eta^{\frac{3}{2} A} \Psi\left(\eta \mathbf{r}_{1}, \ldots, \eta \mathbf{r}_{A}\right),
$$

the plus three energy moment can be expressed by means of the second derivative of the scaled ground-state energy $E_{\text {g.s. }}^{s}(\eta)=\left\langle\Psi_{\eta}|H| \Psi_{\eta}\right\rangle$ as

$$
m_{3}=\frac{1}{2}\left(\frac{2 \hbar^{2}}{M}\right)^{2}\left(\frac{d^{2} E_{\mathrm{g.s.}}^{s}(\eta)}{d \eta^{2}}\right)_{\eta=1} .
$$

Thus the $m_{3}$ moment measures the change of energy of the nucleus when the ground-state wave function is deformed according to (8).

With account of Eqs. (6), (7), and (9), the scaled average energy of the ISGMR is defined as

$$
E_{S}=\sqrt{\frac{m_{3}}{m_{1}}} .
$$

This method allows also to define the scaled nuclear compression modulus for a finite nucleus with mass number $A$ as (see Ref. [12])

$$
K_{A}^{S}=\frac{M}{\hbar^{2}}\left\langle r^{2}\right\rangle_{0} E_{S}^{2}=\frac{1}{A}\left(\frac{d^{2} E_{\text {g.s. }}^{S}(\eta)}{d \eta^{2}}\right)_{\eta=1},
$$

where $M$ is the nucleon mass.

Let us describe the method of calculation of the derivatives in the right-hand sides of Eqs. (9) and (11). Within the QLDFT the scaled energy can be written as

$$
\begin{aligned}
E_{\mathrm{g} . \mathrm{s} .}^{s}(\eta)= & T(\eta)+E_{\mathrm{Nucl}}^{\mathrm{Dir}}(\eta)+E_{\mathrm{Nucl}}^{\mathrm{Exch}}(\eta)+E_{\mathrm{Coul}}(\eta)+E_{\mathrm{so}}(\eta) \\
& +E_{\mathrm{RC}}(\eta) .
\end{aligned}
$$

Due to the fact that in the QLDFT we deal with Slater determinant wave functions, the different contributions to the scaled energy can be easily determined. The kinetic, the spinorbit, and the Coulomb contributions scale as

$$
\begin{gathered}
T(\eta)=\eta^{2} T(1), \quad E_{\mathrm{so}}(\eta)=\eta^{5} E_{\mathrm{so}}(1), \quad \text { and } \\
E_{\mathrm{Coul}}(\eta)=\eta E_{\mathrm{Coul}}(1) .
\end{gathered}
$$

In our approach the residual correlation energy is chosen phenomenologically (see Introduction) as

$$
\begin{aligned}
E_{\mathrm{RC}}[\hat{n}]= & \frac{t_{3}}{4} \int d \mathbf{r} n^{\alpha}(\mathbf{r})\left[\left(2+x_{3}\right) n^{2}(\mathbf{r})-\left(2 x_{3}+1\right)\left(n_{p}^{2}(\mathbf{r})\right.\right. \\
& \left.\left.+n_{n}^{2}(\mathbf{r})\right)\right],
\end{aligned}
$$

which under the scaling transformation reads

$$
E_{\mathrm{RC}}(\eta)=\eta^{3(\alpha+1)} E_{\mathrm{RC}}(1) .
$$

These formulas give a simple way to obtain some of the terms entering (12). Similar explicit expressions for the direct and exchange energy contributions coming from the finite range part of the effective force cannot be derived. However, one can calculate their corresponding derivatives with respect to the dimensionless collective coordinate $\eta$ using the following trick. For the sake of simplicity we will consider a simple Wigner central force. In our approach we use Gogny-like forces with Gaussian form factors $v(r / \mu)$ where $\mu$ is the range of the force. In this case the direct part of the energy coming from the finite range effective force has the general form 


$$
E_{\mathrm{Nucl}}^{\mathrm{Dir}}=\frac{1}{2} \int d \mathbf{r} d \mathbf{r}^{\prime} n(\mathbf{r}) v\left(\left|\mathbf{r}-\mathbf{r}^{\prime}\right| / \mu\right) n\left(\mathbf{r}^{\prime}\right) .
$$

It is easy to see that the scaling of the direct energy is reduced to renormalization of the range $\mu$,

$$
E_{\mathrm{Nucl}}^{\mathrm{Dir}}(\eta)=\frac{1}{2} \int d \mathbf{r} d \mathbf{r}^{\prime} n(\mathbf{r}) v\left(\left|\mathbf{r}-\mathbf{r}^{\prime}\right| /(\eta \mu)\right) n\left(\mathbf{r}^{\prime}\right)=\widetilde{E}_{\mathrm{Nucl}}^{\mathrm{Dir}}(\mu) .
$$

It enables us to write (see Refs. $[13,14]$ )

$$
\begin{aligned}
{\frac{d^{2} E_{\mathrm{Nucl}}^{\mathrm{Dir}}(\eta)}{d \eta^{2}}}_{\eta=1}= & \frac{1}{2} \int d \mathbf{r} d \mathbf{r}^{\prime} n(\mathbf{r})\left[2 s \frac{d v(s / \mu)}{d s}\right. \\
& \left.+s^{2} \frac{d^{2} v(s / \mu)}{d s^{2}}\right] n\left(\mathbf{r}^{\prime}\right),
\end{aligned}
$$

where $s=\mathbf{r}-\mathbf{r}^{\prime}$ is the relative coordinate. In our calculations we, however, used another relation,

$$
\left.\frac{d^{2} E_{\mathrm{Nucl}}^{\mathrm{Dir}}(\eta)}{d \eta^{2}}\right|_{\eta=1}=\left.\mu^{2} \frac{d^{2} \widetilde{E}_{\mathrm{Nucl}}^{\mathrm{Dir}}\left(\mu^{\prime}\right)}{d \mu^{\prime 2}}\right|_{\mu^{\prime}=\mu},
$$

which follows immediately from Eq. (17).

The same is true for the exchange Fock energy $E_{\mathrm{Nucl}}^{\mathrm{Exch}}(\eta)$ coming from the finite range effective force. Our quasilocal exchange energy is obtained by replacing the exact Slater single-particle density matrix by the quasiclassical DM within the extended Thomas-Fermi approximation (ETF) [4]. It is easy to check that the quasiclassical DM $\rho_{\mathrm{ETF}}\left(\mathbf{r}, \mathbf{r}^{\prime}\right)$ $=\rho_{\mathrm{ETF}}(\mathbf{R}, \mathbf{s})$ [with $\mathbf{R}=\left(\mathbf{r}+\mathbf{r}^{\prime}\right) / 2$ ] satisfies the correct scaling transformation law,

$$
\rho_{\mathrm{ETF}}(\mathbf{R}, \mathbf{s}, \eta)=\eta^{3} \rho_{\mathrm{ETF}}(\eta \mathbf{R}, \eta \mathbf{s}) .
$$

Thus, because in the QLDFT the exchange energy has the general form

$$
E_{\mathrm{Nucl}}^{\mathrm{Exch}}=\frac{1}{2} \int d \mathbf{R} d \mathbf{s} \rho_{\mathrm{ETF}}^{2}(\mathbf{R}, \mathbf{s}) v(s / \mu)
$$

we have

$$
E_{\mathrm{Nucl}}^{\mathrm{Exch}}(\eta)=\int d \mathbf{R} d \mathbf{s} \rho_{\mathrm{ETF}}^{2}(\mathbf{R}, \mathbf{s}) v(s /(\eta \mu))=\widetilde{E}_{\mathrm{Nucl}}^{\mathrm{Exch}}(\mu)
$$

and

$$
\left.\frac{d^{2} E_{\mathrm{Nucl}}^{\mathrm{Exch}}(\eta)}{d \eta^{2}}\right|_{\eta=1}=\left.\mu^{2} \frac{d^{2} \widetilde{E}_{\mathrm{Nucl}}^{\mathrm{Exch}}\left(\mu^{\prime}\right)}{d \mu^{\prime 2}}\right|_{\mu^{\prime}=\mu} .
$$

The derivatives in Eqs. (19) and (23) were calculated numerically.

In light nuclei the strength function is much more spread and fragmented than in heavy nuclei (see for instance Ref. [15]). Therefore, to get more insight about the spreading of $S(E)$ we will consider another estimate of the ISGMR mean energy provided by a constrained QLDFT calculation. It is well known (see Ref. [11]) that the $m_{-1}$ RPA sum rule is half the ground-state polarizability with respect to the excitation operator $Q$ of the ISGMR, i.e.,

$$
m_{-1}=\sum_{\nu \neq 0} \frac{|\langle\nu|Q| 0\rangle|^{2}}{E_{\nu}-E_{0}}=-\frac{1}{2}\left(\frac{d R^{2}(\lambda)}{d \lambda}\right)_{\lambda=0}=\frac{1}{2}\left(\frac{d^{2} E_{\text {g.s. }}^{c}(\lambda)}{d \lambda^{2}}\right)_{\lambda=0},
$$

where $R^{2}(\lambda)$ and $E_{\text {g.s. }}^{c}(\lambda)$ are the expectation values of the excitation $(Q)$ and the Hamiltonian $(H)$ operators evaluated with the ground-state wave function of the constrained Hamiltonian $H_{c}=H+\lambda Q$. Using Eqs. (6), (7), and (24), the constrained estimate of the average energy of the ISGMR is defined as

$$
E_{C}=\sqrt{\frac{m_{1}}{m_{-1}}} .
$$

The constrained nuclear compression modulus can also be defined in a similar way to that of Eq. (11):

$$
K_{A}^{C}=\frac{M}{\hbar^{2}}\left\langle r^{2}\right\rangle_{0} E_{C}^{2}=\frac{1}{A}\left(R^{2} \frac{d^{2} \widetilde{E}_{\text {g.s. }}^{c}(R)}{d R^{2}}\right)_{R=R_{0}},
$$

where the function $\tilde{E}_{\text {g.s. }}^{c}(R)$ is defined by the relations $\tilde{E}_{\text {g.s. }}^{c}(R(\lambda))=E_{\text {g.s. }}^{c}(\lambda), R_{0}=R(\lambda=0)$.

As it has been pointed out before, in Ref. [11] was proved that the exact ground-state wave function can be replaced by the HF one in the self-consistent HF+RPA $(1 p 1 h)$ sum rule calculation. Analogous considerations allow to state that the $m_{3}, m_{1}$, and $m_{-1}$ sum rules calculated within QLDFT (i.e., using Slater determinants built up of the single-particle wave functions obtained from the minimization of the QLDFT energy functional), coincide with the QLDFT-based selfconsistent RPA results.

Finally, let us note that the RPA moments fulfill $\sqrt{m_{3} / m_{1}}$ $\geqslant m_{1} / m_{0} \geqslant \sqrt{m_{1} / m_{-1}}$. Therefore, the scaled $\left(E_{S}\right)$ and the constrained $\left(E_{C}\right)$ estimates of the average energy of the resonance give an upper and a lower bound of the mean energy of the ISGMR $m_{1} / m_{0}$. The total RPA width $\sigma$ of the strength distribution can also be estimated from the $E_{S}$ and $E_{C}$ energies:

$$
\sigma \leqslant \frac{1}{2} \sqrt{E_{S}^{2}-E_{C}{ }^{2}} .
$$

\section{NUMERICAL RESULTS}

We have calculated the scaled and the constrained estimates of the average energies of the ISGMR as well as the corresponding compression moduli in finite nuclei using the QLDFT with the D1' [5] and the D1S [6] Gogny forces. In order to test our method we compare in Table I our QLDFT results with the calculations performed in Ref. [8] in the framework of the HF and HF+RPA approaches with the same forces.

It can be seen that the agreement between the constrained QLDFT and HF energies $E_{C}$, and the average HF+RPA energies $\bar{E}_{1,-1}$ is fairly well. The agreement between the scaled 
TABLE I. The ISGMR average energies (in $\mathrm{MeV}$ ) in ${ }^{208} \mathrm{~Pb}$ computed in the framework of the scaled $\left(\bar{E}_{\mathrm{GMR}}^{s}\right)$ and the constrained $\left(\bar{E}_{\mathrm{GMR}}^{c}\right)$ approaches with the D1' and the D1S Gogny forces using the QLDFT compared with the average energies obtained in Ref. [8] using the HF and the HF+RPA methods.

\begin{tabular}{llllll}
\hline \hline & \multicolumn{2}{c}{ QLDFT } & \multicolumn{2}{c}{$\mathrm{HF}$} & \multicolumn{2}{c}{$\mathrm{HF}+\mathrm{RPA}$} \\
& $\bar{E}_{\mathrm{GMR}}^{s}$ & $\bar{E}_{\mathrm{GMR}}^{c}$ & $\bar{E}_{\mathrm{GMR}}^{c}$ & $\bar{E}_{1,-1}$ & $\bar{E}_{3,1}$ \\
\hline $\mathrm{D}^{\prime}{ }^{\prime}$ & 14.51 & 13.93 & 14.05 & 14.15 & 15.33 \\
$\mathrm{D} 1 \mathrm{~S}$ & 13.65 & 13.05 & 13.22 & 13.34 & 14.16 \\
\hline \hline
\end{tabular}

QLDFT energies $E_{S}$ and the values of $\bar{E}_{3,1}$ obtained in HF + RPA approach is reasonable, though it is worse than for the constrained energies. From these comparisons we conclude that using our sum rule approach based on the QLDFT, the corresponding average energies can be confidently used to theoretically estimate the ISGMR energies with Gogny forces, at least for nuclei for which the monopole strength shows a well-defined narrow peak.

In Table II we display results obtained for the nuclei ${ }^{40} \mathrm{Ca}$, ${ }^{90} \mathrm{Zr}$, and ${ }^{208} \mathrm{~Pb}$, for which the experimental excitation energies of the ISGMR are accurately known [7,15].

For the nucleus ${ }^{208} \mathrm{~Pb}$, the QLDFT scaled and constrained predictions of the ISGMR excitation energy computed with the D1' Gogny force agree very well with the experimental centroid value (14.2 MeV). On the other hand, the QLDFT results obtained with the D1S force underestimate the experimental value by a $3.5 \%\left(\bar{E}_{\mathrm{GMR}}^{s}\right)$ and $8 \%\left(\bar{E}_{\mathrm{GMR}}^{c}\right)$. The reason for this discrepancy lies on the fact that the nuclear matter compression modulus for the D1' force $\left(K_{\infty}\right.$ $=228 \mathrm{MeV})$ is larger than that for the D1S force $\left(K_{\infty}\right.$ $=209 \mathrm{MeV})$. The situation is inverse for the ${ }^{90} \mathrm{Zr}$ nucleus where the theoretical QLDFT estimates of the ISGMR energy for D1S force are in a better agreement with the experimental centroid value $(17.9 \mathrm{MeV})$ than that for the $\mathrm{D} 1^{\prime}$ force. Thus $K_{\infty}$ extracted from the sum rule approach using the results for the nucleus ${ }^{208} \mathrm{~Pb}$ is around $230 \mathrm{MeV}$, while the results for the nucleus ${ }^{90} \mathrm{Zr}$ lead to the value around $210 \mathrm{MeV}$, which corresponds to the the upper and lower bounds of the compression modulus predicted in Ref. [8]. For the ${ }^{40} \mathrm{Ca}$ nucleus, the ISGMR energies obtained with our QLDFT plus sum rule approach clearly overestimate the experimental centroid value both for the $\mathrm{D} 1^{\prime}$ and for the D1S forces. In particular, for the D1S force the calculated scaled energy overestimates the $\left(m_{3} / m_{1}\right)^{1 / 2}$ experimental value [15] by a $7 \%$ whereas the constrained energy is larger than the experimental $\left(m_{1} / m_{-1}\right)^{1 / 2}$ by a $21 \%$. For this nucleus our QLDFT model predicts a total RPA width [Eq. (27)] of 3.7 MeV. This means that the monopole strength is quite spread or fragmented and thus the sum rule estimate of the ISGMR for ${ }^{40} \mathrm{Ca}$ should be considered only as qualitative.

The use of radioactive beams allows to obtain nuclei beyond the limits of $\beta$-stability and to reach the neutron drip line for nuclei with $Z \leqslant 8$. Nuclei near the drip line are char-

TABLE II. The compression moduli $K_{A}$ (in MeV) and the ISGMR average energies $\bar{E}_{\mathrm{GMR}}$ (in MeV) of some magic nuclei computed in the framework of the scaled $\left(K_{A}^{s}, \bar{E}_{\mathrm{GMR}}^{s}\right)$ and the constrained $\left(K_{A}^{c}, \bar{E}_{\mathrm{GMR}}^{c}\right)$ approaches with the D1' and the D1S Gogny forces using the QLDFT compared with the same quantities computed with the $\mathrm{SkM}^{*}$ and the SIII Skyrme interactions using the HF approximation. Experimental average energies are taken from Refs. [7,15].

\begin{tabular}{|c|c|c|c|c|c|c|}
\hline & & ${ }^{16} \mathrm{O}$ & ${ }^{28} \mathrm{O}$ & ${ }^{40} \mathrm{Ca}$ & ${ }^{90} \mathrm{Zr}$ & ${ }^{208} \mathrm{~Pb}$ \\
\hline \multirow[t]{4}{*}{$K_{A}^{S}$} & $\mathrm{D} 1^{\prime}$ & 132 & 106 & 146 & 154 & 152 \\
\hline & D1S & 126 & 100 & 137 & 141 & 137 \\
\hline & $\mathrm{SkM}^{*}$ & 124 & 103 & 138 & 146 & 143 \\
\hline & SIII & 199 & 166 & 225 & 243 & 243 \\
\hline \multirow[t]{4}{*}{$\bar{E}_{\mathrm{GMR}}^{s}$} & $\mathrm{D} 1^{\prime}$ & 28.1 & 20.1 & 23.2 & 19.0 & 14.5 \\
\hline & D1S & 27.1 & 19.3 & 22.2 & 18.0 & 13.7 \\
\hline & $\mathrm{SkM}^{*}$ & 26.8 & 19.6 & 22.2 & 18.3 & 13.9 \\
\hline & SIII & 34.5 & 25.3 & 28.5 & 23.4 & 17.9 \\
\hline \multirow[t]{4}{*}{$K_{A}^{c}$} & $\mathrm{D} 1^{\prime}$ & 102 & 14 & 127 & 144 & 140 \\
\hline & D1S & 100 & 14 & 121 & 132 & 125 \\
\hline & $\mathrm{SkM}^{*}$ & 94 & 28 & 119 & 136 & 133 \\
\hline & SIII & 132 & 17 & 178 & 216 & 214 \\
\hline \multirow[t]{4}{*}{$\bar{E}_{\mathrm{GMR}}^{c}$} & $\mathrm{D} 1^{\prime}$ & 24.7 & 7.2 & 21.6 & 18.4 & 13.9 \\
\hline & D1S & 24.1 & 7.3 & 20.9 & 17.5 & 13.1 \\
\hline & $\mathrm{SkM}^{*}$ & 23.2 & 10.2 & 20.6 & 17.6 & 13.3 \\
\hline & SIII & 28.1 & 8.1 & 25.4 & 22.1 & 16.8 \\
\hline $\bar{E}_{\mathrm{GMR}}^{\exp }$ & & & & $19.2 \pm 0.4$ & $17.9 \pm 0.2$ & $14.2 \pm 0.3$ \\
\hline
\end{tabular}


acterized by the small energy of the last bound levels and by their large asymmetry. It is expected that the properties of such nuclei, in particular the ones concerning collective excitations, may considerably differ from the corresponding properties of stable nuclei. Theoretical HF+RPA calculations carried out by Hamamoto, Sagawa, and Zhang [16] using the $\mathrm{SkM}^{*}$ force, have shown that in nuclei near the drip line the distribution of the monopole strength is much affected by the presence of the low-energy threshold due to the tiny bound nucleons. This pattern clearly differs from the one shown by stable nuclei where the strength is concentrated in a single peak at least for medium and heavy nuclei.

In particular the aforementioned authors have analyzed the monopole strength of the ${ }^{28} \mathrm{O}$ nucleus [17]. We have also considered this nucleus in our QLDFT plus sum rule approach and their scaled and constrained energies are also reported in Table II. Although the sum rule approach is unable to deal with fine details of the monopole strength, it provides some signatures about the global behavior of the monopole strength in nuclei near the drip line. In the nucleus ${ }^{28} \mathrm{O}$, the scaled and the constrained energies are clearly reduced with respect to the corresponding values in the stable ${ }^{16} \mathrm{O}$ nucleus, which are also given in Table II. This reduction is particularly significant for the constrained energy and produces a noticeable enhancement of the width of the ISGMR. These facts point out the importance of the low-energy part of the monopole strength in drip line nuclei.

In order to do a complementary check of our QLDFT estimates of the average excitation energies of the ISGMR using the D1' and the D1S forces, we have repeated the sum rule analysis using the Skyrme force $\mathrm{SkM}^{*}$, which has $K_{\infty}$ $=217 \mathrm{MeV}$ slightly larger than the corresponding D1S value $(209 \mathrm{MeV})$. The scaled energies with Skyrme forces are easily calculated [11] and the constrained calculations are performed in the way discussed previously. The excitation energies as well as the finite nuclei incompressibilities obtained with $\mathrm{SkM}^{*}$ are also collected in Table II. It can be seen that D1S and $\mathrm{SkM}^{*}$ practically predicts the same values for the scaled and constrained energies. Actually the excitation energies obtained with $\mathrm{SkM}^{*}$ are slightly larger than the ones computed with D1S, at least for medium and heavy nuclei where the sum rule approach can be regarded more confidently, due to the fact that $K_{\infty}$ is a little bit larger in the former than in the latter force. In the same Table II we also report the ISGMR excitation energies obtained using the Skyrme SIII force which has $K_{\infty}=355 \mathrm{MeV}$. As it has been pointed out in Ref. [8], the energy of the breathing mode is roughly proportional to $\sqrt{K_{\infty}}$. We have checked this behavior with the excitation energies of the ISGMR of the nuclei ${ }^{90} \mathrm{Zr}$ and ${ }^{208} \mathrm{~Pb}$ calculated with the D1S, D1', ${ }^{\prime} \mathrm{SkM}^{*}$, and SIII forces using the scaling method and performing constrained calculations. It is found that this proportionality is also rea- sonably well fulfilled for each nucleus when the QLDFT values are used, especially in the case of the scaled energies.

\section{SUMMARY}

We have analyzed the ability of the recently established quasilocal density functional theory for describing the breathing mode in finite nuclei. First of all, it has to be pointed out that the $1 p 1 h$ RPA calculations based on this theory have similar properties to the HF+RPA calculations. In particular, the exact RPA ground-state wave function can be replaced by the Slater determinants built up of the singleparticle QLDFT wave functions in order to obtain the odd moments of the $1 p 1 h$ RPA strength function. Thus the scaling approach and constrained calculations can be used to obtain the $m_{3}$ and $m_{-1}$ moments (sum rules) of the RPA strength based on the quasilocal density functional theory.

We have applied this sum rule approach using the Gogny forces. For the nucleus ${ }^{208} \mathrm{~Pb}$, we have found an excellent agreement with the HF and the HF+RPA results for the ISGMR reported in the literature. Using the D1' and the D1S Gogny forces and comparing our theoretical estimates of the average energies of the ISGMR with the experimental values of some selected medium and heavy nuclei, we have found that the predicted nuclear matter compression modulus lies in the range $210-230 \mathrm{MeV}$ which also agrees with earlier findings. It is also found that our estimates of the average energies of the ISGMR, in particular the ones obtained with the scaling method, roughly scale with the square root of the compression modulus in nuclear matter.

Although the sum rule approach works basically for medium and heavy stable nuclei where the RPA strength is mainly concentrated in a narrow peak, we have analyzed its predictions in nuclei near the neutron drip line. In this case the RPA strength is broaded and the low energy part considerably enhanced because of the last weakly bound neutrons. The sum rule approach is able to give globally this tendency in such nuclei by reducing the $m_{3}$ and especially the $m_{-1}$ sum rules and thereby increasing the estimate of the resonance width.

\section{ACKNOWLEDGMENTS}

The authors are grateful to Professor S. Shlomo for useful discussions and Dr. M. Centelles for a careful reading of the paper. V.B.S. and V.I.T. would like to acknowledge financial support from the Russian Ministry of Education under Grant No. E02-3.3-463. X.V. acknowledges financial support from DGI (Ministerio de Ciencia y Tecnología and FEDER (Spain) under Grant No. BFM2002-01868 and from DGR (Catalonia) under Grant No. 2001SGR-00064. 
[1] V. B. Soubbotin, V. I. Tselyaev, and X. Viñas, Phys. Rev. C 67, 014324 (2003).

[2] W. Kohn and L. J. Sham, Phys. Rev. 140, A1133 (1965).

[3] P. Hohenberg and W. Kohn, Phys. Rev. 136, B864 (1964).

[4] V. B. Soubbotin and X. Viñas, Nucl. Phys. A665, 291 (2000).

[5] J. Dechargé and D. Gogny, Phys. Rev. C 21, 1568 (1980).

[6] J. F. Berger, M. Girod, and D. Gogny, Comput. Phys. Commun. 63, 365 (1991); Nucl. Phys. A502, 85c (1989).

[7] D. H. Youngblood, H. L. Clark, and Y.-W. Lui, Phys. Rev. Lett. 82, 691 (1999).

[8] J. P. Blaizot, J. F. Berger, J. Dechargé, and M. Girod, Nucl. Phys. A591, 435 (1995).

[9] N. Van Giai, P. F. Bortignon, G. Colò, Z.-M. Ma, and M. R. Quaglia, Nucl. Phys. A687, 44c (2001).
[10] P. Ring and P. Schuck, The Nuclear Many-Body Problem (Springer-Verlag, New York, 1980).

[11] O. Bohigas, A. M. Lane, and J. Martorell, Phys. Rep. 51, 267 (1979).

[12] J. P. Blaizot, Phys. Rep. 64, 171 (1980).

[13] H. Kohno and K. Ando, Prog. Theor. Phys. 61, 1065 (1979).

[14] S. K. Patra, X. Viñas, M. Centelles, and M. Del Estal, Nucl. Phys. A703, 240 (2002).

[15] D. H. Youngblood, Y.-W. Lui, and H. L Clark, Phys. Rev. C 55, 2811 (1997); 63, 067301 (2001).

[16] I. Hamamoto, H. Sagawa, and X. Z. Zhang, Phys. Rev. C 53, 765 (1996); 56, 3121 (1997).

[17] I. Hamamoto and H. Sagawa, Phys. Rev. C 53, 1492 (1996). 